\title{
Aneurysmal Carotid Cavernous Fistula (ACCF), Management and Outcome: Case Report and Review of the Literature
}

\author{
Adil Belhachmi1,2, Yao Christian Hugues Dokponou1,2*\# (), Miloud Gazzaz ${ }^{1,2}$ \\ ${ }^{1}$ Mohammed V University, Rabat, Morocco \\ ${ }^{2}$ Service de Neurochirurgie Hôpital Militaire d'Instruction Mohammed V, Rabat, Morocco \\ Email: abelhachmi31@gmail.com, *dokponou2407@gmail.com, miloudigazzaz29@gmail.com
}

How to cite this paper: Belhachmi, A., Dokponou, Y.C.H. and Gazzaz, M. (2021) Aneurysmal Carotid Cavernous Fistula (ACCF), Management and Outcome: Case Report and Review of the Literature. Open Access Library Journal, 8: e7335.

https://doi.org/10.4236/oalib.1107335

Received: March 21, 2021

Accepted: May 31, 2021

Published: June 3, 2021

Copyright $\odot 2021$ by author(s) and Open Access Library Inc.

This work is licensed under the Creative Commons Attribution International License (CC BY 4.0).

http://creativecommons.org/licenses/by/4.0/

\begin{abstract}
Carotid cavernous fistula is an abnormal communication between the cavernous portion of the carotid artery and the venous bed of cavernous sinus with secondary increased pressure in the cavernous sinus leading to clinical presentations depending on the vascular and/or nervous structures involved in this injury process. The patient may present in the emergency room with proptosis, cranial nerve palsy, increased intraocular pressure, diplopia, decreased vision, and sometimes blindness in case of a direct fistula between the carotid artery and cavernous sinus. This condition is secondary to a traumatic brain injury in the vast majority of cases. A spontaneous rupture of the carotid artery aneurysm into the cavernous sinus is a rare cause of the carotid-cavernous fistula. The aim of this study is to report a case of aneurysmal carotid-cavernous fistula revealed by a spontaneous right pulsatile exophthalmos and its successful management with arterial angiography and endovascular treatment at once.
\end{abstract}

\section{Subject Areas \\ Clinical Medicine}

\section{Keywords}

Carotid-Cavernous Fistula, Aneurysm, Exophthalmos, Endovascular

\section{Introduction}

Carotid cavernous fistulas (CCFs) are abnormal connections between the carotid ${ }^{*}$ Corresponding author.

"Joint first author. 
artery and the cavernous sinus (CS). According to the etiology, they can be traumatic or spontaneous [1]. The spontaneous carotid-cavernous fistulae secondary to an aneurysmal ruptured are rare vascular malformations leading to increased blood pressure in the cavernous bed. Angiographically, CCFs can be classified as direct, when the fistula originates directly in the internal carotid artery (ICA), or indirect when the fistula originates in the dural branches of the carotid artery [1]. Type a fistulas are direct shunts between the internal carotid artery (ICA) and cavernous sinus; Types b, $c$, and $d$ are dural shunts. Type b are fistulas between meningeal branches of the ICA and the cavernous sinus; Type $c$ are dural shunts between meningeal branches of the external carotid artery (ECA) and the cavernous sinus; Type $d$ are those between meningeal branches of both the ICA and ECA and the cavernous sinus (Figure 1) [2]. The great majority of the direct CCFs derive from trauma. Approximately a quarter of these lesions occur spontaneously, generally caused by the rupture of an intracavernous aneurysm [3].

Even though the diagnosis can be clinically easily suspected with sudden headache, exophthalmos, and/or presence of bruit in the eyeball, an emergency neurosurgical treatment is uncommon and quite difficult due to the topography of the cavernous sinus. The implication of the interventional neuroradiological procedures, these last two decades, in the management of carotid-cavernous fistula have been lifesaving for patients mostly when done in emergency settings, and help to avoid vital complications such as blindness, subarachnoid hemorrhage, or intracerebral hematomas. We report a case of aneurysmal carotid-cavernous fistula revealed by a spontaneous left pulsatile exophthalmos and its successful management by endovascular treatment.

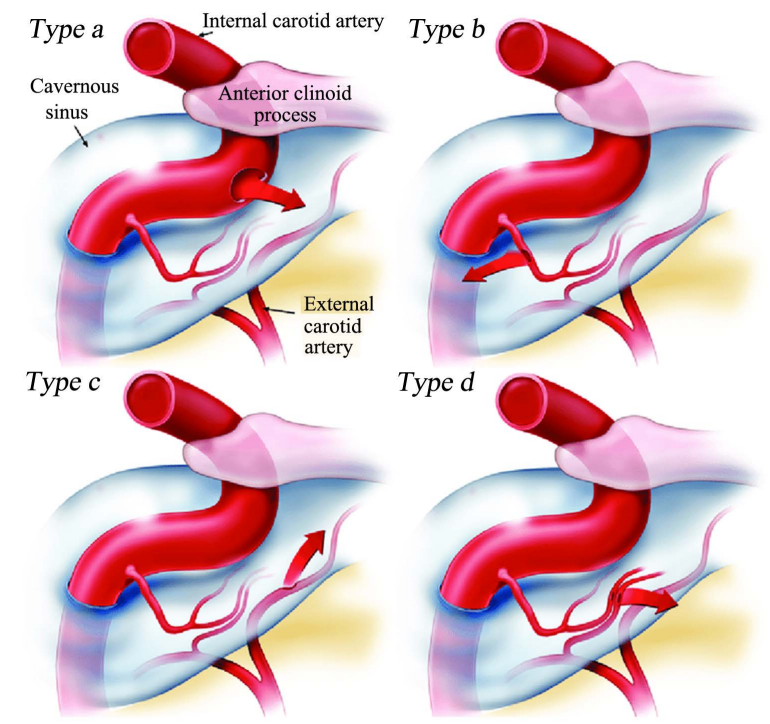

Figure 1. Barrow classification of CCFs. Type a fistulas are characterized by direct shunting of blood flow from the ICA into the cavernous sinus. Type $b$ and $c$ fistulas are shunts to the cavernous sinus from branches of the ICA and ECA, respectively. Type $d$ fistulas have shunts from both the ICA and ECA simultaneously. Ellis et al. [20]. 


\section{Case Presentation}

A 48-year-old female housewife with no past medical history was admitted to the emergency room at Mohammed V Military Teaching Hospital of Rabat for sudden and severe fronto-orbital headache, blurry vision, and right exophthalmia ongoing for the past forty-eight hours. The patient denied any history of trauma prior to the appearance of symptoms. She declares no other medical problems. Her familial, surgical, and gynecological history was unremarkable.

On physical and neurological examination, she was conscious and well oriented in time and space; a little bit anxious, holding her forehead in the hands and unable to close the right eye with obvious eyeball protrusion. The eyeball was red, hyperemic, presence of peribulbar arterialized loops with murmurs (bruit) overheard when the stethoscope is put on the eyelid. There was no fever, and no sensitive or motor palsy.

\subsection{Investigations}

The non-symmetrical presentation of the cavernous sinus on the CT-Scan was more revealing a possible carotid-cavernous lesion with a round hyperdense lesion on the right carotid-cavernous sinus helping to conclude of either aneurysmal rupture or a vascular malformation bleed (Figure 2(a)). The cerebral MRI revealed a unilateral right carotid-cavernous fistula secondary to the aneurysmal rupture (Figure 2(b)). The patient was then transferred to the neurological surgery department for further investigations and treatment. Urgent cerebral arteriography was done the same day by interventional neuroradiologists, showing right high-flow CCF, Barrow Type A from a ruptured right internal carotid artery aneurysm, venous hypertension of CS, and ipsilateral ectasia of the superior ophthalmic vein (Figure 3(a)). The arterial blood pressure, ECG, pulmonary $\mathrm{X}$-Ray, Complete blood count, and blood coagulation factors were without abnormal findings.

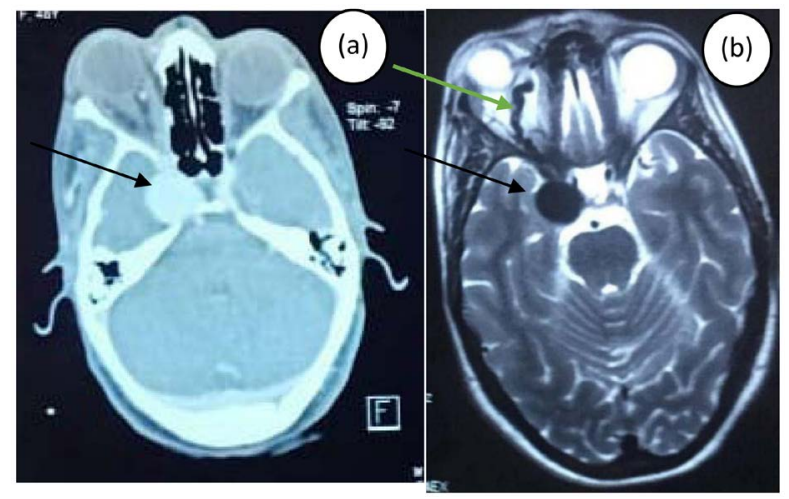

Figure 2. (a) Contrast enhanced brain computer tomography, axial section on parenchymal window, showing a round hyperdense lesion on the right carotid-cavernous sinus (black arrow). Extraocular muscle thickening and periorbital oedema. (b) Brain MRI, axial T2-weighted sequence with spontaneous high flow unilateral right carotid-cavernous fistula secondary to the aneurysmal rupture; ectasia of right ophthalmic vein (green arrow). 


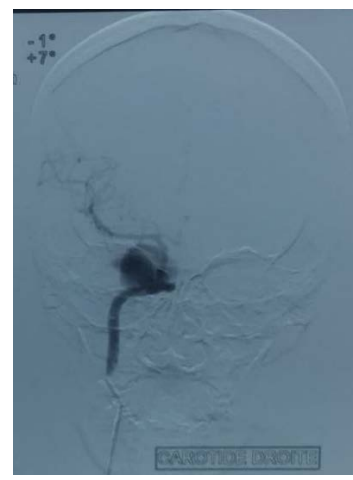

(a)

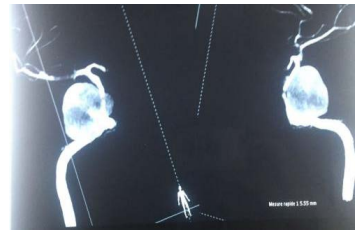

(b)

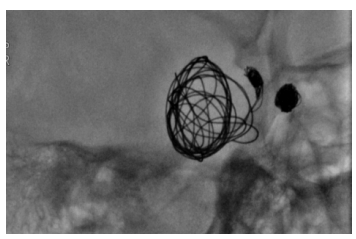

(c)

Figure 3. (a) Right carotid-cavernous fistulas secondary to aneurysmal rupture. (b) Endovascular treatment with positioning detachable balloons and coils into the right cavernous sinus. (c) Immediate post-op control.

\subsection{Management}

During that same diagnosis procedure and through the same right femoral artery access, the fistula was catheterized with a microcatheter, interrupting the arterio-venous communication. Endovascular treatment was then performed positioning detachable balloons and coils into the right cavernous sinus. Immediate postoperative control confirmed total occlusion of the fistula (Figure $3(\mathrm{~b})$ \& Figure 3(c)).

\subsection{Outcome and Follow-Up}

Two days after the endovascular treatment, the headaches are subsided, the vision becomes better with a progressive regression of the exophthalmos. The patient recovered and was discharged on day 5 post-operative with medical treatment. She came back (three) 3 months later for follow-up and was doing well (Figure 4).

\section{Discussion}

The cavernous sinus is defined as a dural envelope through which the intracranial extradural segment of the internal carotid artery (ICA) and its branches traverse from the petrous and lacerum segments to the interdural and intradural supraclinoid segments. An abnormal communication between the ICA and external carotid artery (ECA) or any of their branches and the cavernous sinus is termed a carotid-cavernous fistula (CCF) [4]. The triad of exophthalmos, epibulbar arterialized loops, and blurry vision couple with the "bruit" of the bulging eyeball should be enough for physicians in the emergency room or in the ICU to suspect a diagnosis of the carotid-cavernous fistula [5].

The imaging modality of choice for suspected cavernous sinus lesions is the MRI with and without contrast offering higher soft-tissue contrast resolution compared to $\mathrm{CT}$ and the protocol should include routine $\mathrm{T} 2$, fluid-attenuated inversion recovery (FLAIR), and precontrast T1-weighted images of the entire brain. Postcontrast T1-weighted 3-mm-thick images should be obtained in the 


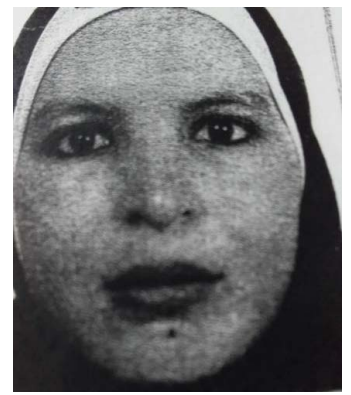

(a)

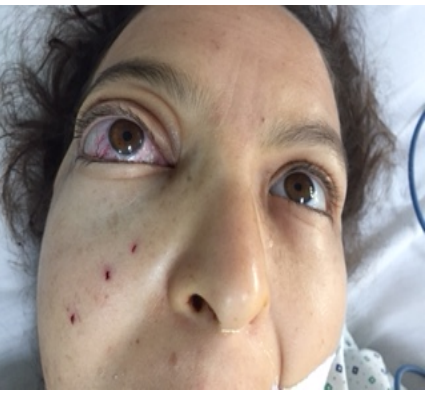

(b)

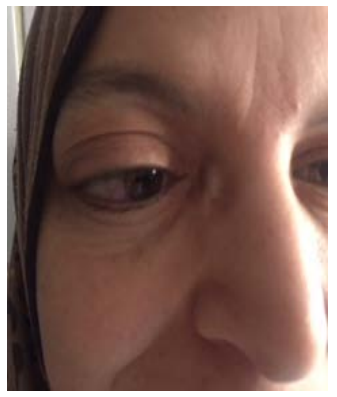

(c)

Figure 4. (a) Before ACCF. (b) Right eye exophthalmos secondary to the CCF. (c) Regression of exophthalmos 5 days after endovascular treatment.

axial and coronal planes with at least 1 plane imaged with a fat-saturation technique. CT or MR angiography may be helpful when vascular pathology such as carotid-cavernous fistula (CCF) or ICA aneurysm is suspected [6] [7] [8] [9]. In our study, is MR imaging that reveals the diagnosis with modifications found in the right cavernous sinus (Figure 2). Cerebral digital subtraction angiography (DSA) is the gold standard radiological investigation for any CCF clinically suspected and leading to its endovascular management.

Classification of carotid-cavernous fistulas (CCFs) into the four types described by Barrow allows the surgeon to choose the optimal therapy for each patient. Type A patients have fast flow fistulas that are manifested by a direct connection between the internal carotid arterial siphon and the cavernous sinus through a single tear in the arterial wall. The best therapy is the obliteration of the connection by a detachable balloon [10]. In this same series of 132 cases of CCFs, three cases were ruptured cavernous aneurysms and were treated with endovascular balloon detachable coiling as well as Ninety-two of 95 traumatic CCFs. The remaining 3 patients had undergone unsuccessful surgical trapping. Several authors have reported successful microsurgical treatment of cavernous aneurysms and CCFs since Parkinson and later Dolenc described a direct approach to these lesions. Concurrently, endovascular techniques evolved so that these lesions could be treated with selective aneurysmal occlusion or parent artery occlusion with detachable balloons. During an 11-year period, Bavinzski et al. [11] successfully treated 32 patients with cavernous aneurysms by endovascular techniques by means of microcatheters and detachable balloons. They reported excellent clinical results with obliteration by embolization of cavernous aneurysms managed with detachable coils becoming an important endovascular tool [12] [13] [14] [15]. Thus, this management becomes the cornerstone whenever these aneurysms happen to rupture into the cavernous sinus.

Endovascular embolization is the preferred treatment for carotid-cavernous fistulas (CCFs), but failure to catheterize the cavernous sinus may occur as a result of tortuosity, hypoplasia, or stenosis of the normal venous routes. Wolfe et al. [16] reviewed the records of 10 patients with indirect CCF, previously undergone unsuccessful endovascular attempts at treatment at their institution. As 
endovascular techniques for treatment of direct CCFs continue to evolve by detachable balloons or coils to occlude the shunt while sparing the carotid artery, liquid embolic agents are being lately used with promising good result, and Zenteno et al. [17] have described complete occlusion after embolization of CCF with a novel approach using Onyx as the sole embolic material. This patient's life has been spars thanks to the availability and accessibility of endovascular procedure for diagnosis and treatment at once. Physicians of emergency filed need not only to rise their awareness and be updated about this kind of life threatening conditions, but also need to be trained and be prepared for such patient management.

\section{Conclusion}

Arterial embolization in emergency settings should be thought in front of any patient presenting the striking diagnostic triad (exophthalmos, epibulbar arterialized loops, and blurry vision) coupled with the bruising. Failing to do so might leave the patient with severe complications from visual impairment to blindness.

\section{Ethics and Reporting Guidelines}

Informed consent and verbal permission were obtained from the patient prior to the submission of this article. Also, this article respects both the Consensus-based Clinical Case Reporting Guideline and the Recommendations for the Conducting, Reporting, Editing, and Publication of Scholarly Work in Medical Journals [18] [19].

\section{Conflicts of Interest}

The authors declare not having any conflict of interest in this case report and there are no financial resources.

\section{References}

[1] de Aguiar, G.B., Jory, M., de Almeida Silva, J.M., Conti, M.L.M. and Veiga, J.C.E. (2016) Advances in the Endovascular Treatment of Direct Carotid-Cavernous Fistulas. Revista da Associação Médica Brasileira, 62, 78-84. https://doi.org/10.1590/1806-9282.62.01.78

[2] Barrow, D.L., Spector, R.H., Braun, I.F., Landman, J.A., Tindall, S.C. and Tindall, G.T. (1985) Classification and Treatment of Spontaneous Carotid-Cavernous Sinus Fistulas. Journal of Neurosurgery, 62, 248-256. https://doi.org/10.3171/jns.1985.62.2.0248

[3] Luo, C.-B., Teng, M.M.-H., Chang, F.-C., Lirng, J.-F. and Chang, C.-Y. (2004) Endovascular Management of the Traumatic Cerebral Aneurysms Associated with Traumatic Carotid Cavernous Fistulas. American Journal of Neuroradiology, 25 501-505.

[4] Ringer, A.J., Salud, L. and Tomsick, T.A. (2005) Carotid Cavernous Fistulas: Anatomy, Classification, and Treatment. Neurosurgery Clinics, 16, 279-295. https://doi.org/10.1016/j.nec.2004.08.004

[5] Keizer, R. (2003) Carotid-Cavernous and Orbital Arteriovenous Fistulas: Ocular 
Features, Diagnostic and Hemodynamic Considerations in Relation to Visual Impairment and Morbidity. Orbit, 22, 121-142. https://doi.org/10.1076/orbi.22.2.121.14315

[6] Razek, A.A. and Castillo, M. (2009) Imaging Lesions of the Cavernous Sinus. American Journal of Neuroradiology, 30, 444-452.

https://doi.org/10.3174/ajnr.A1398

[7] Munawar, K., Nayak, G., Fatterpekar, G.M., Sen, C., Zagzag, D., Zan, E., et al. (2020) Cavernous Sinus Lesions. Clinical Imaging, 68, 71-89. https://doi.org/10.1016/j.clinimag.2020.06.029

[8] Go, J.L. and Rajamohan, A.G. (2017) Imaging of the Sella and Parasellar Region. Radiologic Clinics of North America, 55, 83-101.

[9] Jindal, G., Miller, T., Raghavan, P. and Gandhi, D. (2017) Imaging Evaluation and Treatment of Vascular Lesions at the Skull Base. Radiologic Clinics of North America, 55, 151-166.

[10] Debrun, G.M., Viñuela, F., Fox, A.J., Davis, K.R. and Ahn, H.S. (1988) Indications for Treatment and Classification of 132 Carotid-Cavernous Fistulas. Neurosurgery, 22, 285-289. https://doi.org/10.1227/00006123-198802000-00001

[11] Bavinzski, G., Killer, M., Ferraz-Leite, H., Gruber, A., Gross, C.E. and Richling, B. (1998) Endovascular Therapy of Idiopathic Cavernous Aneurysms over 11 Years. American Journal of Neuroradiology, 19, 559-565.

[12] Cohen, J.E., Gomori, J.M., Segal, R., Spivak, A., Margolin, E., Sviri, G., et al. (2008) Results of Endovascular Treatment of Traumatic Intracranial Aneurysms. Neurosurgery, 63, 476-486. https://doi.org/10.1227/01.NEU.0000324995.57376.79

[13] Debrun, G., Lacour, P., Vinuela, F., Fox, A., Drake, C.G. and Caron, J.P. (1981) Treatment of 54 Traumatic Carotid-Cavernous Fistulas. Journal of Neurosurgery, 55, 678-692. https://doi.org/10.3171/jns.1981.55.5.0678

[14] Gupta, A.K., Purkayastha, S., Krishnamoorthy, T., Bodhey, N.K., Kapilamoorthy, T.R., Kesavadas, C., et al. (2006) Endovascular Treatment of Direct Carotid Cavernous Fistulae: A Pictorial Review. Neuroradiology, 48, 831-839. https://doi.org/10.1007/s00234-006-0132-X

[15] van Rooij, W.J., Sluzewski, M. and Beute, G.N. (2006) Ruptured Cavernous Sinus Aneurysms Causing Carotid Cavernous Fistula: Incidence, Clinical Presentation, Treatment, and Outcome. American Journal of Neuroradiology, 27, 185-189.

[16] Wolfe, S.Q., Cumberbatch, N.M., Aziz-Sultan, M.A., Tummala, R. and Morcos, J.J. (2010) Operative Approach via the Superior Ophthalmic Vein for the Endovascular Treatment of Carotid Cavernous Fistulas That fail Traditional Endovascular Access. Operative Neurosurgery, 66, ons293-ons299.

https://doi.org/10.1227/01.NEU.0000369705.91485.38

[17] Zenteno, M., Santos-Franco, J., Rodríguez-Parra, V., Balderrama, J., Aburto-Murrieta, Y., Vega-Montesinos, S., et al. (2010) Management of Direct Carotid-Cavernous Sinus Fistulas with the Use of Ethylene-Vinyl Alcohol (Onyx) Only: Preliminary Results. Journal of Neurosurgery, 112, 595-602.

https://doi.org/10.3171/2009.6.JNS09440

[18] Gagnier, J.J., Kienle, G., Altman, D.G., Moher, D., Sox, H., Riley, D., et al. (2013) The Care Guidelines: Consensus-Based Clinical Case Reporting Guideline Development. Case Reports, 2013, Article ID: bcr2013201554. https://doi.org/10.1136/bcr-2013-201554

[19] International Committee of Medical Journal Editors (2019) Recommendations for the Conducting, Reporting, Editing, and Publication of Scholarly Work in Medical 
Journals. International Committee of Medical Journal, 1-19.

http://www.icmje.org/recommendations/

[20] Ellis, J.A., Goldstein, H., Connolly, E.S. and Meyers, P.M. (2012) Carotid-Cavernous Fistulas. Neurosurgical Focus FOC, 32, E9.

https://doi.org/10.3171/2012.2.FOCUS1223 